\title{
Absolute Positionsbestimmung mit MR-Sensoren an einspurigen Maßkörpern
}

\author{
Dipl.-Ing. Bogdan Kneißig, Dipl.-Ing. Andreas Voss, Dipl.-Ing. Armin Meisenberg, Dipl.- \\ Ing. Reinhold Pieper, Dr. Axel Bartos \\ Buisness Unit Magnetic Sensors \\ MEAS-Deutschland GmbH, Hauert 13, 44227 Dortmund \\ Contact: Andreas.Voss@meas-spec.com, 02319740560
}

\section{Kurzfassung}

Magnetoresistive Positionssensoren dienen der Messung linearer Bewegungen, indem die wechselnde Magnetisierung einer magnetischen Skala mit einem MR-Sensor abgetastet wird. Durch den Einsatz von mehreren zueinander versetzten MR-Sensoren und einem einspurigen magnetischen Maßkörper mit einer in der Pollänge codierten Positionsinformation ist eine absolute Positionsbestimmung möglich.

\section{Abstract}

Position sensors based on the magnetoresistance effect combine high precision with cost effectiveness for linear displacement measurements. The use of magnetic scales has some advantages over optical encoders, as they can be used in many environments. Single track scales with modified pole pitch geometries allow the precise and contactless measurement of absolute positions with extended range in limited available space.

\section{Einleitung}

Das Erfassen von Positionsinformationen wird durch eine Vielzahl von Sensorkonzepten abgedeckt. Weglängensensoren, die auf dem anisotropen magnetoresistiven Effekt basieren, vereinen die Vorteile einer hochpräzisen und wirtschaftlichen Lösung zur Messung der Positionsinformation in einem weiten Temperaturbereich. Durch die kontaktlose Messung ist es möglich, die Systeme auch unter anspruchsvollen Einsatzbedingungen zu verwenden, da die benötigten Komponenten z.B. chemisch geschützt und faktisch verschleißfrei arbeiten können. Zudem ist eine hohe Toleranz gegen Verschmutzung sichergestellt.

Magnetoresistive Absolutmesssysteme sind bisher aus zwei- oder mehrspurigen Skalen [1] und den dazugehörigen Sensoren aufgebaut, wobei eine Polspur inkremental vermessen wird und die restlichen Spuren zur groben Lokalisation der Absolutposition verwendet werden. Die Abdeckung größerer Weglängen macht dabei immer breiter werdende Magnetskalen sowie eine erhebliche Anzahl an Sensoren notwendig.

Daneben wurden auch einspurige Absolutsysteme [3] vorgeschlagen, die mit minimaler Sensoranzahl Verfahrwege bis zu $100 \mathrm{~mm}$ hochpräzise abdecken können.

\section{AMR-Technologie}

AMR(anisotrop Magnetoresistiv)-Winkelsensoren bestehen im Allgemeinen aus mäanderförmig angeordneten Widerstandsstreifen, welche zu Wheatstonebrücken verschaltet sind. Durch diese Verschaltung kann die Ausgangsspannung $U_{a}$ einer Sensor-Brücke in Abhängigkeit zum externen Feldwinkel $\Theta$ wie folgt geschrieben werden: Ein extern angelegtes magnetisches Feld richtet die Magnetisierung der Widerstandsstreifen so aus, dass der Magnetisierungsvektor dem Vektor des externen Feldes nahezu parallel folgt [2] wobei ein $\cos ^{2}$-Zusammenhang zwischen Widerstand und externem Feldwinkel $\Theta$ besteht (vgl. Gl.1). Da es sich hier um Starkfeldsensoren handelt, muss das 
externe Feld $H_{0}$ hoch genug sein um die Felddrehung der Widerstandsstreifen in Sättigung zu betreiben. Ein schwächeres Feld führt zu Genauigkeitsverlust, da nicht alle Domänen korrekt ausgerichtet sind und so der widerstandsbestimmende Effekt nur verfälscht auftritt.

$$
U_{a}(\Theta) \cong \frac{U_{b}}{2} k_{A M R} \cdot \sum_{n=2,6,10} A_{n} \cos (n \cdot(\Theta-\alpha)) \quad(G l .1)
$$

Standard AMR-Winkelsensoren verwenden zwei gegeneinander verschobene, ineinander verschachtelte Brücken (vgl. Abb. 1), um die für die Signalauswertung erforderlichen, mit $180^{\circ}$ periodischen sin- und cosAusgangssignale zu erzeugen. Form und Lage der Mäander tragen dazu bei, dass das Ausgangssignal von Oberwellen der 3. und 5. Ordnung ( $n=6,10$; vgl. Gl.2) befreit wird.
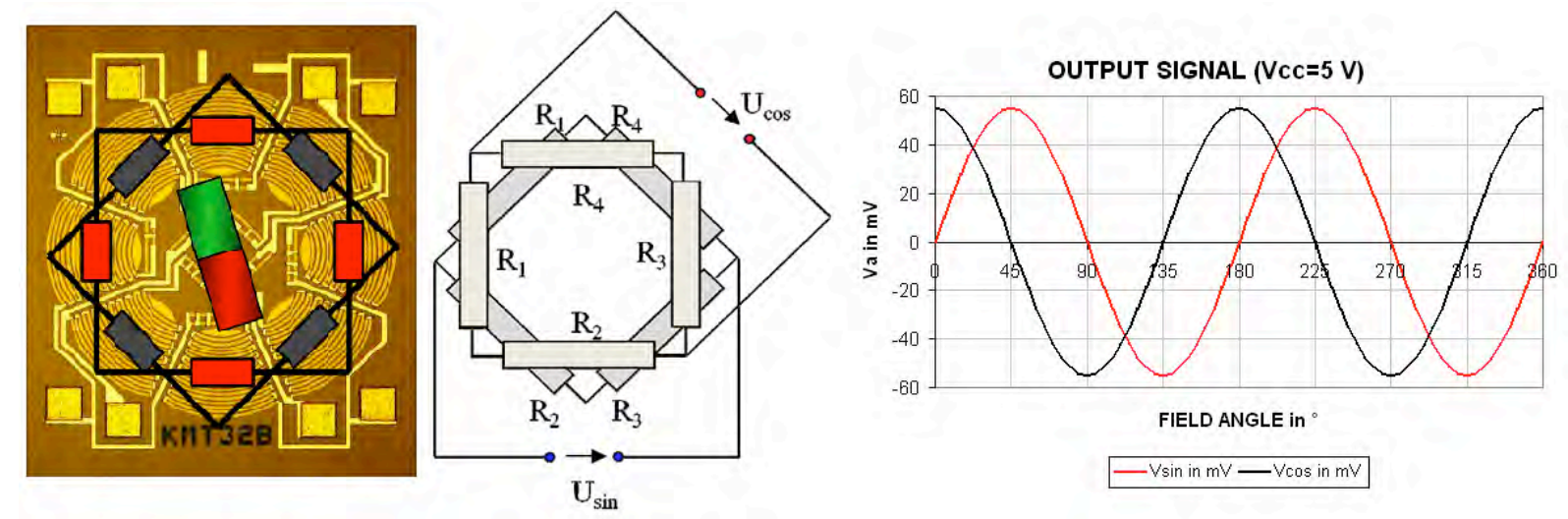

Abbildung 1 Sensorlayout, Verschaltungsprinzip und Signalausgang eines KMT32B [4]

Als feldgebende Struktur wird ein mit definierten Pollängen $p_{0}$ magnetisierter Maßstab verwendet. Das dadurch entstehende nahezu kreisförmige magnetische Feld, welches vektoriell im Bereich von $0^{\circ}$ bis $180^{\circ}$ pro Pol dreht, wird von den Sensoren linear in Maßstabsrichtung gescannt.

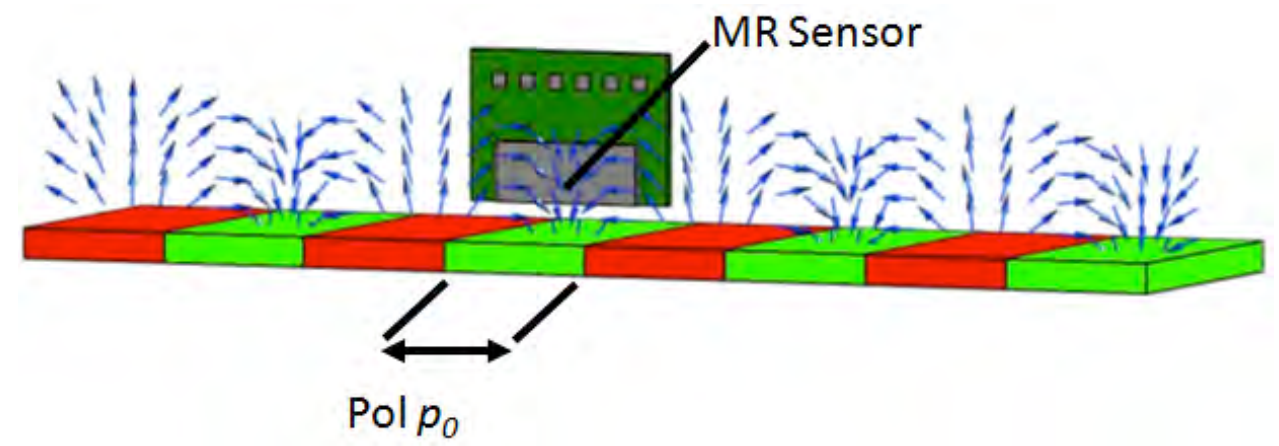

Abbildung 2 Magnetskala und Messprinzip

Durch den Aufbau der MR-Sensoren aus miteinander als Wheatstonebrücken verschalteten magnetisch sensitiven Widerständen kann die Ausgangsspannung $U_{a}$ in Abhängigkeit von der Position $x$ einer Sensor-Brücke in erster Näherung wie folgt geschrieben werden [1],

$$
U_{a}=k_{A M R} \cdot \sin \left(\pi \cdot \frac{x}{p_{0}}\right)
$$

wobei $p_{0}$ die Länge eines Poles auf dem magnetischen Maßkörper beschreibt. Beide Messbrücken sind dabei so angeordnet, dass sich um $90^{\circ}$ versetzte Signale ergeben. Daher werden die Ausgangssignale 
auch als $U_{\sin }$ und $U_{\text {cos }}$ bezeichnet. Durch die Verkettung beider Signale in eine Arcus-Tangens-Funktion entsteht ein linearer Zusammenhang zwischen dem vom Sensor "gesehenen" magnetischem Feldwinkel $\Theta$ und den resultierenden Sensorsignalen. Wie in GI.3 zu sehen ist, handelt es sich hierbei um eine reine Verhältnisrechnung, welche mögliche Temperatureffekte elegant eliminiert.

$$
\Theta=\frac{1}{2} \arctan \left(\frac{U_{\text {sin }}}{U_{\cos }}\right) \quad(G l .3)
$$

Als Werkstoff für AMR-Sensoren kommt hauptsächlich das ungiftige Permalloy [6] zum Einsatz. Neben einer sehr kleinen Koerzitivfeldstärke (Hk < 1) zeichnet es sich durch das Fehlen von Magnetostriktion und somit eine große Unempfindlichkeit gegen mechanische Einflüsse aus. Die Herstellung von AMR Sensoren ist ein etablierter Prozess wobei der einschichtige Aufbau von AMR-Sensorstrukturen vielfältige Möglichkeiten zur Anpassung des Sensordesigns an die gewählte Messaufgabe bietet. Als Maß für die Widerstandsänderung eines AMR-Sensorelementes in Abhängigkeit eines externen gerichteten Feldes wird der AMR-Koeffizient $k_{A M R}$ definiert. Er stellt eine Materialkonstante dar und liegt für Permalloy im Bereich von $2-3 \%$ für Winkeländerungen von 0 bis $90^{\circ}$ zur Messstromrichtung im Sensorelement.

\section{Einspurig absolut und genau}

Die Grundidee des hier vorgestellten einspurigen linearen Absolutmesssystems basiert auf Pseudo Random Codes [5] und der Vorgabe, dass an jedem Punkt der Positionsmessung ein eindeutiges Bitmuster zu Grunde liegt. Die Magnetskala ist z.B. mit einem 8-Bit langen Pseudo-Random-Code (Kettencode) [5] in der Pollänge absolut kodierten. Unter einem Kettencode versteht man eine sich wiederholende Folge (Kette) von Zufallszahlen, wobei die binäre Information 0 und 1 mit sich unterscheidenden Pollängen in der Magnetskala codiert wird. Die Anzahl der verwendeten Zufallszahlen ist von der Bitbreite des Codes abhängig.

Im vorliegenden Konzept wird ein Absolutmesssystem durch eine Magnetskala mit variierenden Pollängen und einer Sensoranordnung, bestehend aus z.B. 16 um die Abstände $d_{n}$ zueinander versetzten MR-Sensoren aufgebaut. Damit sind $2^{8}=256$ zufällige Zahlenkombinationen möglich, was bei einer Basispollänge von $p_{0}=4,9 \mathrm{~mm}$ zu einer absolut detektierbaren Maximallänge von $d_{\max }=1254 \mathrm{~mm}$ führt.

Die MR-Sensoren messen den lokalen Feldwinkelverlauf über der Magnetskala, anhand dessen anschließend auf die zugehörige absolute Position über der Skala geschlossen werden kann. Jede BitKombination ist auf der Skala nur einmalig vorhanden bis sie sich wiederholt. Daraus folgt, dass jede Bitkombination positionsbezogen eindeutig zurückführbar (absolut kodiert) ist. Abb.2 zeigt einen Ausschnitt der verwendeten Magnetskala und der 16 Sensoren.

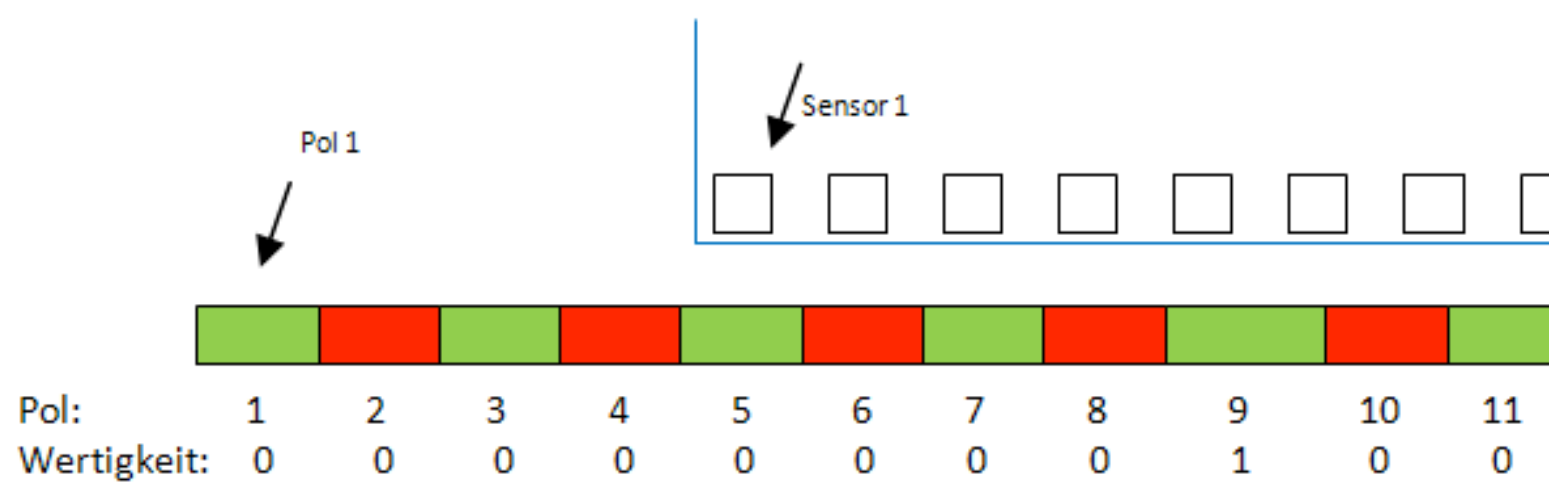

Abbildung 3 Prinzip der Sensoranordnung 
Des Weiteren ist die Polnummer und die dazugehörige Wertigkeit der detektierten Bit-Kombination zu erkennen. Die binären Wertigkeiten der unterschiedlichen Bit-Kombinationen werden durch unterschiedlich lange Nord- und Süd-Pole (Polbreiten) auf dem Polstreifen realisiert.

Bei einer punktuellen Messung wird zunächst mit Hilfe der 16 Sensoren jeder dazugehörige Feldwinkel $\varphi_{n}$ berechnet. Dies erfolgt durch einer Arcus-Tangens-Auswertung, wobei das Verhältnis der Brückenausgangssignale ein Maß für den Feldwinkel $\varphi_{n}$ an der aktuellen Position der Sensoren $(n=1-16$ für Sensor 1 bis Sensor 16) ist.

$$
\varphi_{n=1-16}=\frac{1}{2} \arctan \left(\frac{U_{n, \sin }}{U_{n, \cos }}\right)
$$

Der Feldwinkel $\varphi_{n}$ selbst gibt zwar Aufschluss über die Position des Sensors über dem aktuellen Pol, jedoch fehlt die Information über welchem Pol gerade gemessen wird. Dies bedeutet, dass nicht bekannt ist über welcher Code-Kombination die 16 Sensoren sich aktuell befinden.

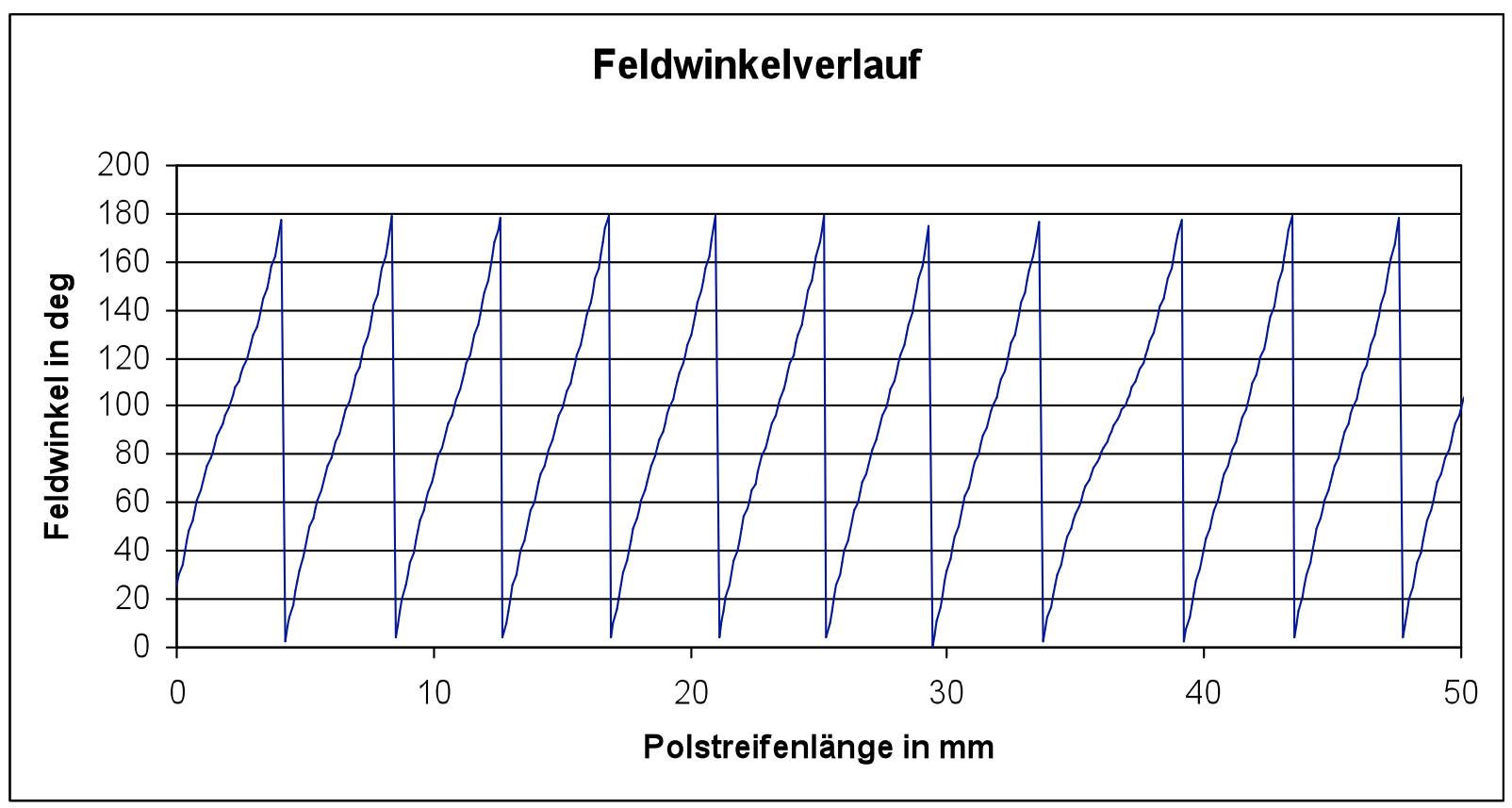

\section{Abbildung 4 Feldwinkelverlauf über Magnetskala}

Die relativen Abstände der Polgrenzen der Magnetskala zueinander ermöglichen diese Zuordnung (Abb.4). Hierzu werden mittels der ermittelten Feldwinkel $\varphi_{n}$, Winkel liegt zwischen 0 und $180^{\circ}$, die daraus resultierenden relativen Positionen der Polgrenzen zwischen den Sensoren extra- und interpoliert (Abb.5). 


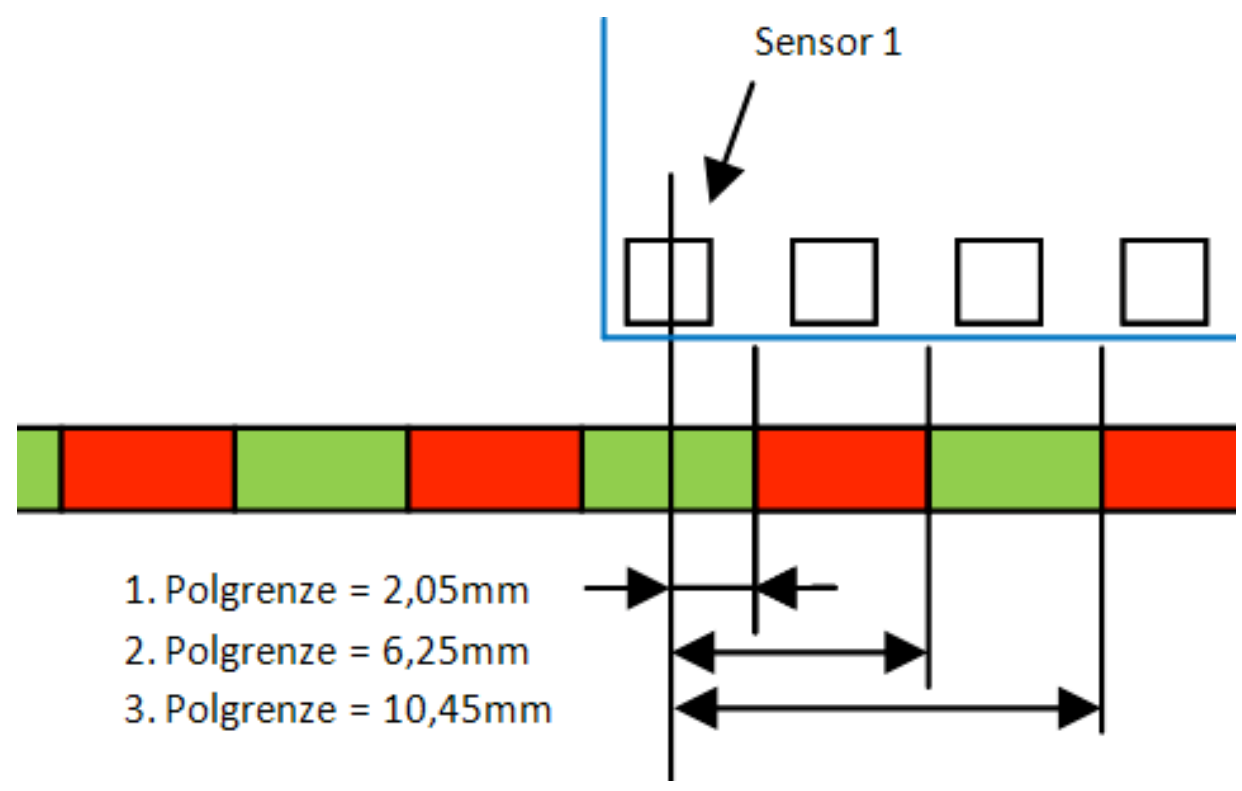

Abbildung 5 Relative Positionen der Polgrenzen

Durch diese relativen Positionsangaben kann schließlich auf die aktuellen Polbreiten (Abstände zwischen den Polgrenzen) geschlossen werden, welche wiederum eine Codekombination des absolut kodierten Kettencodes widerspiegeln. Mit dem ermittelten Code kann anschließend aus einer Lookup-Tabelle die aktuelle absolute Position auf der Magnetskala ermittelt werden.

$$
\text { Polbreite }_{n=1-16}=\text { Polgrenze }_{n+1}-\text { Polgrenze }_{n} \quad(G l .5)
$$

Die Bestimmung der Absolutposition kann nochmals verbessert werden, indem bei der Berechnung des Feldwinkels die tatsächliche Polbreite am jeweiligen Sensorort berücksichtigt wird. Eine Look-Up-Table, welche die Geometrie der verwendeten Magnetskala enthält, führt ebenfalls zur Steigerung der Messgenauigkeit, da auf diesen Weg systembedingte Abweichungen aufgrund der gewählten analytischen Näherung entfallen.

\section{Leistungsfähigkeit des Systems}

Dem System liegt die Idee zu Grunde, dass mit nur einem einspurig absolut kodierten magnetischen Maßkörper beliebig lange Messstrecken realisiert werden können. Dabei variiert lediglich die Bitanzahl des verwendeten Kettencodes und die Anzahl der Sensoren um eine Bitkombination des Codes zu erkennen. Beim Entwurf des Systems wurde darauf geachtet, dass bei dem verwendeten magnetischen Maßkörper eine geeignete Polbreitenkombination verwendet wird, die ein sicheres Detektieren der Polbreiten und den daraus resultierenden Kettencode ermöglicht. Beispielsweise kann mit 16 Sensoren und einem 8-Bit langen Kettencode eine Strecke von bis zu 1,25m vermessen werden. 


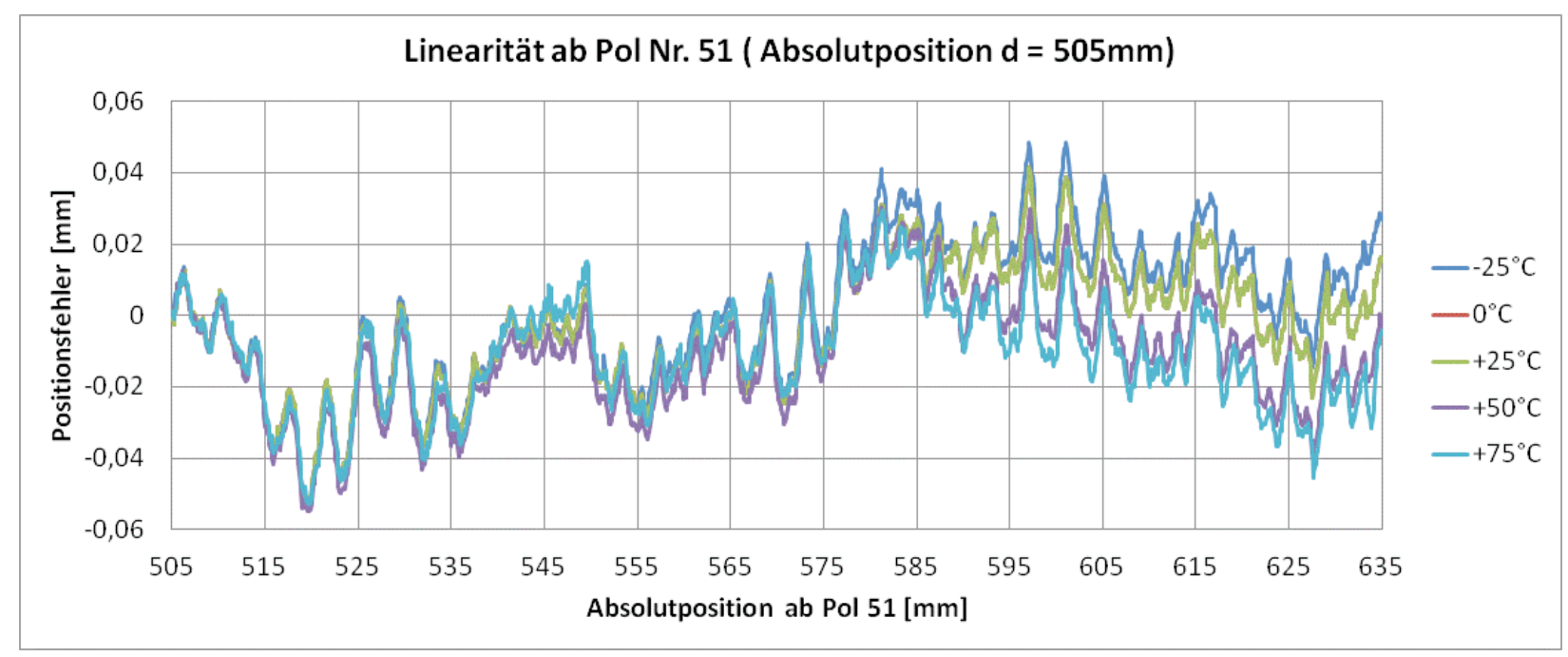

Abbildung 6 Linearität eines Beispielsystems mit $2^{8}=256$ Polen zur Polbasis $5 \mathrm{~mm}$ ab Pol Nr. 51

Bei der Konzeptionierung des Systems muss dabei darauf geachtet werden, dass bei dem verwendeten Polstreifen eine geeignete Polbreitenkombination verwendet wird, die ein sicheres detektieren der Polbreiten und den daraus resultierenden Kettencode ermöglicht. Bei einer schlecht gewählten Polbreitenkombination werden die ermittelten Polbreiten (Wertigkeiten der Pole) fasch erkannt, was zu einer falschen absoluten Position führt.

Da lediglich der Feldwinkel über der Magnetskala zur Ermittlung des Kettencodes benötigt wird, kann für die Messung des Feldwinkels auf Standardelemente wie z.B. dem KMT32B [4] (Drehwinkelsensor) zurückgegriffen werden, dessen Signalverlauf mit speziellen Kalibrieralgorithmen auf den Feldverlauf über linearen magnetischen Maßkörpern angepasst wird.

Die Absolutposition kann nur in dem Bereich ermittelt werden, in dem sich alle Sensoren des Systems über dem Maßkörper befinden.

Trotz der relativ hohen Anzahl an Sensoren lässt sich das System sehr kompakt und kostengünstig aufbauen. Das hier vorgestellte Absolutsystem mit 16 MR Sensoren kommt mit einer Lesekopflänge von $80 \mathrm{~mm}$ aus.

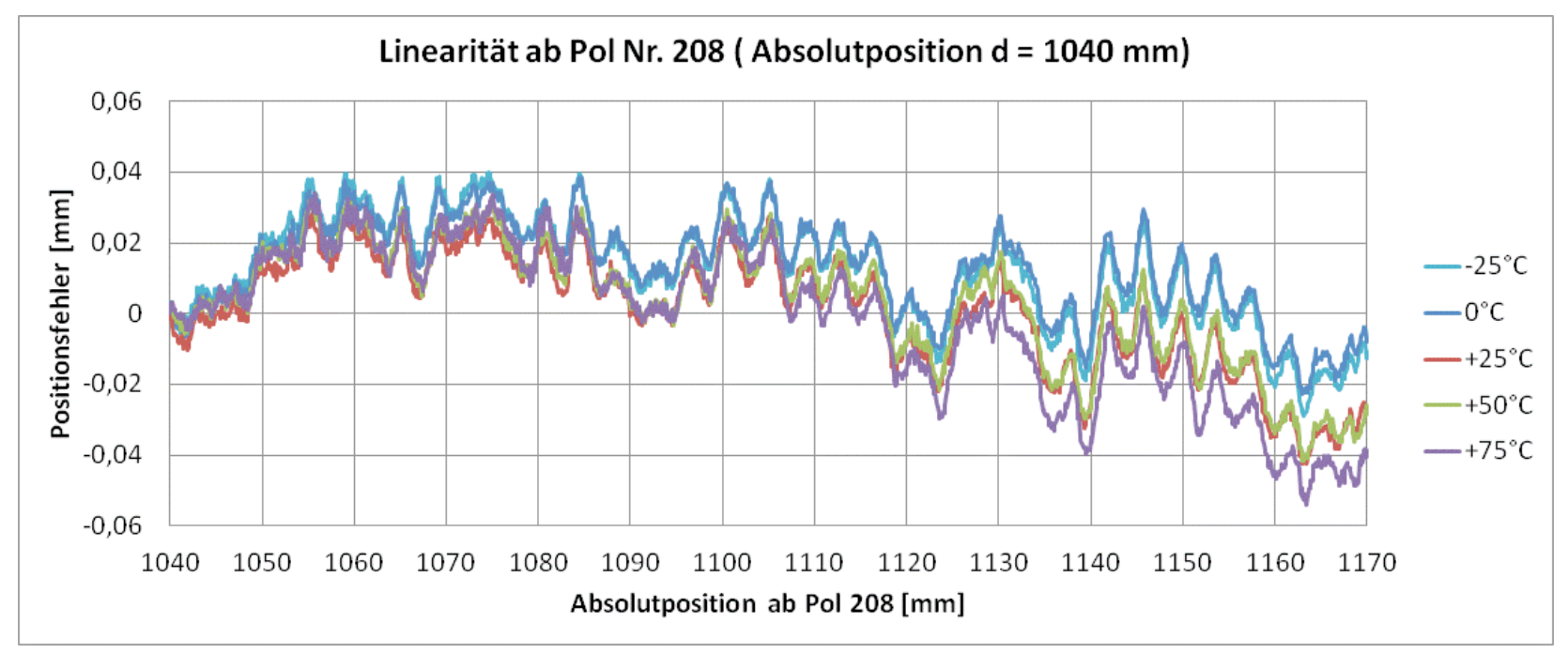

Abbildung 7 Linearität eines Beispielsystems mit $2^{8}=256$ Polen zur Polbasis $5 \mathrm{~mm}$ ab Pol Nr. 208

Aufgrund der einfachen Erweiterbarkeit auf längere Distanzen bietet sich das vorgestellte System zur absoluten Messung sehr langer Strecken an, wie in der nachfolgenden Tabelle gezeigt: 


\begin{tabular}{|c|c|c|}
\hline $\begin{array}{l}\text { Kettencode } \\
\text { mit N - Bits }\end{array}$ & $\begin{array}{l}\text { Max. Magnetskalen- } \\
\text { länge in Meter }\end{array}$ & $\begin{array}{l}\text { Mindestanzahl von Sensoren um eine Bit- } \\
\text { kombination zu erkennen (ohne Redundanz) }\end{array}$ \\
\hline 2 & 0,0196 & 4 \\
\hline 3 & 0,0392 & 6 \\
\hline 4 & 0,0784 & 7 \\
\hline 5 & 0,1568 & 8 \\
\hline 6 & 0,3136 & 10 \\
\hline 7 & 0,6272 & 11 \\
\hline 8 & 1,2544 & 13 \\
\hline 9 & 2,5088 & 14 \\
\hline 10 & 5,0176 & 15 \\
\hline 11 & 10,0352 & 17 \\
\hline 12 & 20,0704 & 18 \\
\hline
\end{tabular}

Tabelle 1 Minimalanforderungen an ein Kettencodesystem

Durch die Verwendung einer schmalen Polspur entfallen Probleme mit magnetischen Wechselwirkungen, wie sie bei Mehrspursystemen auftreten können. Damit benötigt das System einen kleinen Bauraum bei sehr präziser Positionsauswertung mit einer Genauigkeit von wenigen Mikrometern, was mit aktuellen inkrementelle Positionssensoren vergleichbar ist. Die Komplexität und auch die Kosten der einspurigen magnetischen Skala sinken im Vergleich zu absolut messenden Mehrspuranwendungen deutlich.

\section{Literatur}

[1] A.Schütz "MR-Sensoranwendungen in der Automatisierungstechnik“ in: 8. Symposium magnetoresistive Sensoren, Wetzlar, 2005

[2] Klaus C.J. Dietmayer (2001). Magnetische Sensoren auf Basis des AMR-Effekts (Magnetic Sensors Based on the AMR-Effect) tm -Technisches Messen: Vol. 68, Issue 6, pp. 269

[3] A.Voss „Absolute Positionsbestimmung mit magnetoresistiven Sensoren“ in Sensor und Messsysteme 2008 ISBN 978-3-18-092011-

[4] KMT32B Magnetischer Drehwinkelsensor [www.meas-spec.com]

[5] US-Patent Nr. 2632058 vom 17.03.1952

[6] McGuire T.R.: Potter R.T.: Anisotropic magnetoresistance in ferromagnetic 3d alloys. IEEE Transactions on Magnetics, Vol. Mag-11, No- 4, (7/1975) 